\title{
Characterization of a Giardia lamblia WB C6 clone resistant to the isoflavone formononetin
}

\author{
Maaike Sterk,† Joachim Müller,† Andrew Hemphill and Norbert Müller \\ Institute of Parasitology, University of Berne, Länggass-Strasse 122, CH-3012 Berne, Switzerland
}

Correspondence

Norbert Müller

nmueller@ipa.unibe.ch

Joachim Müller

joachim.mueller@ipa.unibe.ch

Received 25 May 2007

Revised 29 August 2007

Accepted 7 September 2007

\begin{abstract}
Giardia lamblia is a common intestinal-dwelling protozoan and causes diarrhoea in humans and animals worldwide. For several years, a small number of drugs such as the 5-nitroimidazole metronidazole (MET) or the thiazolide nitazoxanide (NTZ) have been used for chemotherapy against giardiasis. However, various pre-clinical and clinical investigations revealed that antigiardial chemotherapy may be complicated by emergence of giardial resistance to these drugs. The present study addressed the question if isoflavones with antigiardial activity, such as daidzein (DAl) or formononetin (FOR), may serve as alternative compounds for treatment of giardiasis. For this purpose, the potential of G. lamblia clone WB C6 to form resistance to FOR and related isoflavones was tested in vitro. In the line of these experiments, a clone (C3) resistant to isoflavones, but sensitive to MET and NTZ, was generated. Affinity chromatography on DAl-agarose using cell-free extracts of $\mathrm{G}$. lamblia trophozoites resulted in the isolation of a polypeptide of approximately $40 \mathrm{kDa}$, which was identified by mass spectrometry as a nucleoside hydrolase (NH) homologue (EAA37551.1). In a nucleoside hydrolase assay, recombinant $\mathrm{NH}$ hydrolysed all nucleosides with a preference for purine nucleosides and was inhibited by isoflavones. Using quantitative RT-PCR, the expression of genes that are potentially involved in resistance formation was analysed, namely $\mathrm{NH}$ and genes encoding variant surface proteins (VSPs, TSA417). The transcript level of the potential target $\mathrm{NH}$ was found to be significantly reduced in C3. Moreover, drastic changes were observed in VSP gene expression. This may indicate that resistance formation in Giardia against isoflavones is linked to, and possibly mediated by, altered gene expression. Taken together, our results suggest FOR or related isoflavones as an alternative antigiardial agent to overcome potential problems of resistance to drugs like MET or NTZ. However, the capacity of Giardia to develop resistance to isoflavones can potentially interfere with this alternative treatment of the disease.
\end{abstract}

\section{INTRODUCTION}

Giardia lamblia can cause (human) giardiasis, which is a notorious health problem throughout the world (reviewed by Thompson, 2000). Infection by this parasite is one of the most important causes of diarrhoea in the world, and is especially prevalent in children in developing countries. Giardiasis is considered as a disease of the poor and has been included in the 'Drugs for Neglected Diseases Initiative' list (Savioli et al., 2006). This initiative is focused, among others, on the urgent need for effective drugs with minor side effects. In the past, 5-nitroimidazole metronidazole (MET) (commercially known as Flagyl) and a few other drugs such as thiazolide nitazoxanide (NTZ)

†These authors equally contributed to this work.

Abbreviations: DAl, daidzein; FOR, formononetin; GEN, genistein; MET, 5-nitroimidazole metronidazole; NH, nucleoside hydrolase; NTZ, thiazolide nitazoxanide; PDI, protein disulfide isomerase; VSPs, variant surface proteins. (commercially known as Alinia) have been used as therapy against giardiasis (reviewed by Gardner \& Hill, 2001; Upcroft \& Upcroft, 2001). Although these compounds are able to efficiently kill Giardia trophozoites, treatment of giardiasis with these drugs may be associated with recurrence of symptoms (e.g reviewed by Upcroft \& Upcroft, 2001). Furthermore, drug resistance is one of the reasons for treatment failure. Resistance to MET and other nitroimidazoles has mostly been demonstrated or induced in vitro, but various genotypically distinct isolates with reduced drug susceptibility have also been isolated from human patients (reviewed by Upcroft \& Upcroft, 2001; Upcroft et al., 1999, 2006). In contrast, clinical isolates resistant to NTZ have not yet been found. However, our recent in vitro studies revealed that exposure of G. lamblia trophozoites to increasing concentrations of NTZ resulted in resistance formation, and at least one of the highly resistant clones isolated exhibited cross-resistance to MET (Müller et al., 2007). This observation suggests a careful re-evaluation of NTZ regarding its 
suitability as an alternative drug for treatment of METresistant giardiasis.

In order to improve the situation in chemotherapy of resistant giardiasis, new potential pharmaceuticals from natural sources - such as isoflavones isolated from the bark of Dalbergia frutescens - have been evaluated regarding their antigiardial activity in vitro (Khan et al., 2000). Isoflavones are mainly found in Leguminosae where they have anti-oxidant, anti-microbial and signalling functions (reviewed by Dakora \& Phillips, 1996; Dixon \& Steele, 1999). Within this group of compounds, Khan et al. (2000) identified formononetin (FOR), a major isoflavone of Ononis sp., as the most potent antigiardial agent (Fig. 1). In vitro, FOR has an $\mathrm{IC}_{50}$ value more than 5 times lower than MET, and more than 150 times lower than genistein (GEN), a major isoflavone of soybean (Khan et al., 2000). Furthermore, even at high concentrations this compound did not exhibit a cytotoxic effect in a Vero cell culture and had no detectable adverse effects in mice (Khan et al., 2000). Due to the observations that FOR and MET have differential kinetics of antigiardial activity in vitro and possess a differential spectrum of antiprotozoal activity, the mode of action of these two drugs was suggested to be completely different (Khan et al., 2000).

It is evident that an eventual chemotherapeutical application of FOR against giardiasis can be complicated by the capacity of Giardia to develop resistance to this alternative drug. In the present study, we have established an in vitro cultivation assay to find out if FOR treatment against giardiasis promotes formation of antigiardial drug resistance. In particular, we have generated FOR-resistant trophozoites by increasing stepwise the amount of FOR in the culture and have characterized a resistant clone with respect to growth in the presence of isoflavones, NTZ and MET. In order to identify isoflavone-binding proteins, we have developed an affinity chromatography approach (see Müller et al., 2007) coupling the simplest isoflavone, daidzein (DAI) to agarose and succeeded in isolating a polypeptide of approximately $40 \mathrm{kDa}$, which was identified by mass spectrometry as a nucleoside hydrolase $(\mathrm{NH})$ homologue (EAA37551.1). The resistant clone was

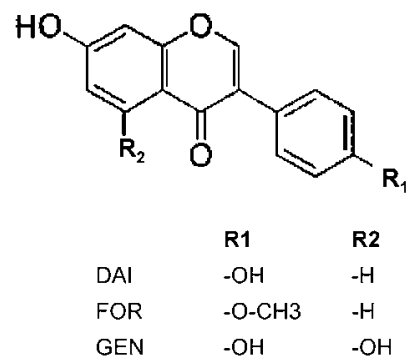

Fig. 1. Structural formulas of isoflavones used in this study. DAl, daidzein; FOR, formononetin; GEN, genistein. analysed regarding $\mathrm{NH}$ gene expression. Furthermore, since upon selective drug pressure G. lamblia trophozoite populations may undergo antigenic variation (Müller et al., 2007), variant surface protein (VSP) gene expression was also analysed in this study.

\section{METHODS}

Biochemicals and drugs. If not otherwise stated, all biochemical reagents including the isoflavones FOR, GEN and DAI were from Sigma. For structural formulas see Fig. 1. They were kept as $100 \mathrm{mM}$ (FOR, GEN) or $40 \mathrm{mM}$ (DAI) stock solutions in DMSO at $4{ }^{\circ} \mathrm{C}$.

Drug treatment assays. Trophozoites from G. lamblia WB C6 were grown under anaerobic conditions in $10 \mathrm{ml}$ culture tubes (Nunc) containing modified TYI-S-33 medium as described (Keister, 1983). The effects of drugs in axenic G. lamblia trophozoite cultures were assayed as previously described (Müller et al., 2007).

Generation of FOR-resistant Giardia lamblia trophozoites. FOR-resistant G. lamblia trophozoites were generated using parasites with the same genetic background (i. e. WB C6). In order to obtain drug resistant trophozoites, G. lamblia WB C6 wild-type parasites grown to confluency were initially cultured in the presence of sublethal concentrations (100 nM) of FOR. After 3 days, the medium was removed, viable trophozoites remained attached to the tube walls, and fresh medium was added, containing $250 \mathrm{nM}$ FOR. Living (attached) trophozoites were counted under the microscope as previously described (Müller et al., 2006). When the respective numbers remained constant or increased, fresh medium was added, containing similar or slightly higher FOR concentrations. In case the number of living trophozoites decreased, medium was replaced by fresh medium without the corresponding drug (see Megraud et al., 1998). This operation was repeated during eight passages until levels of $500 \mathrm{nM}$ FOR were reached. Clones from the resistant cell line were generated by two rounds of limited dilution of trophozoites (Müller et al., 2007). In total, 12 clones having maintained their resistance were subcultivated. Among these clones, clone 3 (C3) exhibited the shortest generation time and no visible morphological alterations in the presence of the drug, and was selected for detailed characterization. C3 was kept in long-term cultures by periodic passaging in modified TYI-S-33 medium containing $500 \mathrm{nM}$ FOR.

Preparation of crude extracts from G. Iamblia trophozoites. Crude extracts were prepared by suspending $10^{8}$ trophozoites in $2 \mathrm{ml}$ extraction buffer, i.e. PBS containing $0.1 \%$ Triton X-100 and $1 \mathrm{mM}$ phenyl-methyl-sulfonyl fluoride followed by vigorous vortexing and centrifugation $\left(10000 \mathrm{~g}, 4{ }^{\circ} \mathrm{C}\right)$. The pellet was re-extracted once with $2 \mathrm{ml}$ and once with $1 \mathrm{ml}$ of extraction buffer. The supernatants were pooled, yielding approximately $5 \mathrm{ml}$ of crude extract.

Affinity chromatography using DAl-agarose. In order to produce DAI-agarose, $0.75 \mathrm{~g}$ lyophilized epoxy-agarose with a C-12 spacer was suspended in $15 \mathrm{ml} \mathrm{H}_{2} \mathrm{O}$ and centrifuged at $300 \mathrm{~g}$ for $5 \mathrm{~min}$. Washes in water were repeated twice, and once using coupling buffer $\left(\mathrm{NaHCO}_{3} 0.1 \mathrm{M}, \mathrm{pH} 9.5\right)$. After the last wash, $20 \mathrm{mg}$ DAI was added and coupling buffer was added to a maximum volume of $5 \mathrm{ml}$. The mixture was incubated for 3 days at $37{ }^{\circ} \mathrm{C}$ under slow but continuous shaking in order to allow coupling of the epoxy group to DAI via one of its $\mathrm{OH}$ groups. The resulting column medium (approx. $2 \mathrm{ml}$ ) was then transferred to a chromatography column (Novagen, Merck) and the column was washed with coupling buffer $(20 \mathrm{ml})$. This was followed by ethanolamine $(1 \mathrm{M}, \mathrm{pH} 9.5)$ for $4 \mathrm{~h}$ at $20^{\circ} \mathrm{C}$ in the absence of light in order to block residual reactive groups. Finally, the column was extensively washed with PBS and PBS/DMSO $(1: 1)$ in 
order to remove unbound DAI. The DAI column was stored in PBS containing $0.02 \% \mathrm{NaN}_{3}$ at $4{ }^{\circ} \mathrm{C}$.

Prior to affinity chromatography, the DAI column was washed with $50 \mathrm{ml}$ PBS equilibrated at $20{ }^{\circ} \mathrm{C}$. Crude extracts $(5 \mathrm{ml})$ of Giardia trophozoites prepared as described above were loaded with a flow rate of approximately $0.25 \mathrm{ml} \mathrm{min}{ }^{-1}$. The column was washed with PBS until the baseline was flat ( 8 column volumes, corresponding to about $24 \mathrm{ml}$ ). Proteins binding to the DAI column were eluted with $0.1 \mathrm{mM}$ FOR in PBS followed by elution with a pH shift (glycine $\mathrm{Cl}^{-}$ $100 \mathrm{mM}, \mathrm{pH} 2.9)$ in order to remove non-specifically bound proteins. Moreover, fractions were taken before elutions with FOR (pre-FOR) or $\mathrm{pH}$ shift (pre-pH shift). Sizes of these fractions ranged between 3 and $5 \mathrm{ml}$. From all fractions, $0.05-0.2 \mathrm{ml}$ aliquots were taken for analysis by SDS-PAGE. SDS-PAGE was performed according to Laemmli (1970) using a Hoefer Minigel 250 apparatus (Amersham). Silver staining was performed according to Blum et al. (1987).

Protein sequencing by mass spectrometry. For protein sequencing, the FOR eluates with the highest amounts of binding protein were pooled and dialysed against ammonium bicarbonate $\left(1 \mathrm{~g}^{-1}\right)$ for $4 \mathrm{~h}$, then against $0.4 \mathrm{~g} \mathrm{l}^{-1}$ overnight at $4{ }^{\circ} \mathrm{C}$ in the dark. The dialysed fraction was then lyophilized. Aliquots of the lyophilized binding protein (approx. $200 \mathrm{ng}$ ) were suspended in SDS-PAGE sample buffer, loaded on a $12 \%$ acrylamide gel and subjected to electrophoresis. After staining with colloidal Coomassie $(0.1 \%$ Coomassie brilliant blue G 250 in $34 \%$ methanol with $0.5 \%$ acetic acid and $17 \%$ ammonium sulfate), a band of approximately $40 \mathrm{kDa}$ was excised and processed for mass spectrometry analysis performed by Core Facility Proteomics (Centre Médical Universitaire, Geneva, Switzerland).

Cloning and heterologous expression of Giardia lamblia nucleoside hydrolase (NH). In order to clone $\mathrm{NH}$ into the Histag-expression vector pET151 directional TOPO (Invitrogen), a forward (NHfullF; see Table 1) and reverse (NHfullR, see Table 1) primer (MWG Biotech) were created for the amplification of gene fragments encoding the $\mathrm{NH}$ polypeptide. CACC at the $5^{\prime}$ end of NHfullF was added in order to allow directional cloning. The cyclic $\mathrm{NH}$ gene amplification reaction, cloning of the $\mathrm{NH}$ gene amplification product into pET151 directional TOPO, as well as bacterial expression and subsequent His-tag purification of recombinant $\mathrm{NH}$ was done according to the procedure previously applied for production of recombinant protein disulfide isomerase (PDI) 4 of G. lamblia WB clone C6 (Müller et al., 2007). Purified recombinant G. lamblia NH protein $\left(\right.$ recGlNH) was stored in $50 \%$ glycerol at $-20{ }^{\circ} \mathrm{C}$.
NH enzyme assay. To determine $\mathrm{NH} K_{\mathrm{m}}$ value, activity was measured by a direct assay based on the hydrolysis of inosine (Parkin et al., 1991). The assay was performed in a quartz cuvette containing HEPES $50 \mathrm{mM}, \mathrm{pH} 7.3$ with inosine in concentrations ranging from 0 to $2 \mathrm{mM}$ in a dual-beam spectrophotometer (UV 1700; Shimadzu). After initiation of the reaction by adding recGlNH ( 1 to $5 \mu \mathrm{M}$ ), absorption was continuously read at $280 \mathrm{~nm}$. The values were corrected by a corresponding substrate blank inserted into the second beam. The values obtained were fitted to the Lineweaver-Burk transformation of the Michaelis-Menten law in order to calculate the $K_{\mathrm{m}}$.

For the determination of substrate specificity and for inhibition studies, recGlNH was assayed using an indirect colorimetric assay of the ribose released based on the cuproin method as described previously (Parkin et al., 1991). Eppendorf tubes containing $0.1 \mathrm{ml}$ HEPES $50 \mathrm{mM}$, pH 7.3 with substrates and/or isoflavones as indicated were incubated for $30 \mathrm{~min}$ to $1 \mathrm{~h}$ at $37{ }^{\circ} \mathrm{C}$ in a water bath. Reaction was stopped by adding $0.4 \mathrm{ml}$ water, $0.15 \mathrm{ml}$ cuproin reagent $\mathrm{I}\left(\mathrm{Na}_{2} \mathrm{CO}_{3} 4 \%\right.$, glycine $\left.1.6 \%, \mathrm{CuSO}_{4} \cdot 5 \mathrm{H}_{2} \mathrm{O} 0.05 \%\right)$ and $0.15 \mathrm{ml}$ cuproin reagent II (cuproin, 2,9-dimethyl-1,10-phenanthrolin $0.12 \%$ suspended in water) followed by $10 \mathrm{~min}$ incubation at $95{ }^{\circ} \mathrm{C}$. After cooling to room temperature, absorption was measured at $450 \mathrm{~nm}$. Blanks for enzyme, substrate and isoflavones were included and subtracted from the reaction. A standard curve (glucose from 0 to $100 \mathrm{nmol}$ ) made in HEPES buffer under reaction conditions was included in each assay.

Processing of RNA samples and quantitative reverse transcription (RT)-PCR. For quantification of gene expression by realtime RT-PCR, trophozoites of wild-type WB C6 and isoflavone resistant clone $\mathrm{C} 3$ were grown in the absence of drugs until near confluency was reached. Cells were harvested as described, and RNA was extracted using a Qiagen RNeasy kit (Qiagen) and including a DNase I digestion (to remove residual genomic DNA) according to the instructions provided by the manufacturer. RNA was eluted with $50 \mu \mathrm{l}$ RNase free water and stored at $-80^{\circ} \mathrm{C}$.

First-strand cDNA synthesis using a polyT-ANC primer (von Allmen et al., 2004; primer sequences, see Table 1) and subsequent quantitative ACT-, VSPtot- and TSA417-PCR (primer sequences, see Table 1) were done as previously described (Müller et al., 2007). In parallel runs, forward (NHquantF) and reverse (NHquantR) primers (see Table 1) were applied for the quantitative NH-PCR. From the quantitative RT-PCR, mean values ( \pm SEM) from triplicate determinations were assessed and expression levels of $\mathrm{NH}$, VSPtot and TSA417 were given as values in arbitrary units relative to the constitutively expressed 'house keeping' gene ACT.

Table 1. Overview of primers used in this study

CDS, coding sequence; utr, untranslated.

\begin{tabular}{|c|c|c|}
\hline Gene Accession number & Region of CDS & Primer $\left(5^{\prime} \rightarrow 3^{\prime}\right)$ \\
\hline Actin (ACT) EAA39190 & CDS 715-933 & $\begin{array}{l}\text { ACTquantF ACATATGAGCTGCCAGATGG; } \\
\text { ACTquantR TCGGGGAGGCCTGCAAAC }\end{array}$ \\
\hline Nucleoside hydrolase (NH) EAA37551.1 & $\begin{array}{l}\text { CDS } 1-1097 \text { plus } 7 \text { of } 3^{\prime} \text { utr } \\
\text { CDS } 751-1037\end{array}$ & $\begin{array}{l}\text { NHfullF CACCATGGCTGTCACAAAGCAC; } \\
\text { NHfullR GAATTCACTATTTCGTTAGG; } \\
\text { NHquantF GGGCGCTTCCACCGATC; } \\
\text { NHquantR GAATTCACTATTTCGTTAGGGT }\end{array}$ \\
\hline Variant surface protein TSA417 U89152.1 & CDS $127-335$ & $\begin{array}{l}\text { TSA417quantF TGTGGAACGTGTGCCAATAG; } \\
\text { TSA417quantR AGACACGTAGTACAGTCGG }\end{array}$ \\
\hline Variant surface protein (total) & see von Allmen et al. (2004) & $\begin{array}{l}\text { VSP }_{\text {tot }} \text { quantF (MM16) GGCTTCCTCTGCTGGTGGTTC; } \\
\text { VSP }_{\text {tot }} \text { quantR (ANC) GACCACGCGTATCGATGTCGA }\end{array}$ \\
\hline
\end{tabular}


Statistical methods. The significance of the differences of the NH, VSPtot and TSA417 gene, and gene expression between wild-type clone WB C6 and isoflavone resistant clone C3 were determined by Student's $t$-test using Microsoft's Excel program. $P$ values of $<0.01$ were considered statistically highly significant.

\section{RESULTS}

\section{Generation of FOR-resistant (FORr) Giardia lamblia trophozoites}

By axenic culture of G. lamblia WB C6 trophozoites in the presence of slowly and continuously increasing concentrations of FOR (see Methods), a drug-resistant trophozoite line was generated. This trophozoite line was subcultured in the presence of up to $500 \mathrm{nM}$ FOR. In total, 12 clones from the resistant cell line were generated and one FORr clone (C3) was further investigated. Regarding the growth kinetics in normal medium, clone $\mathrm{C} 3$ did not significantly differ from wild-type clone WB C6 (Fig. 2). Upon prolonged growth of $\mathrm{C} 3$ in drug-free medium for more than 10 generations, resistance was maintained. The clone C3 encysted as well as the wild-type when put on encystation medium (not shown).

\section{Cross-resistance}

In order to determine $\mathrm{IC}_{50}$ values of the $\mathrm{FORr}$ clone $\mathrm{C} 3$ in the presence of FOR and the related isoflavone DAI, trophozoites of the respective strains were grown for 3 days

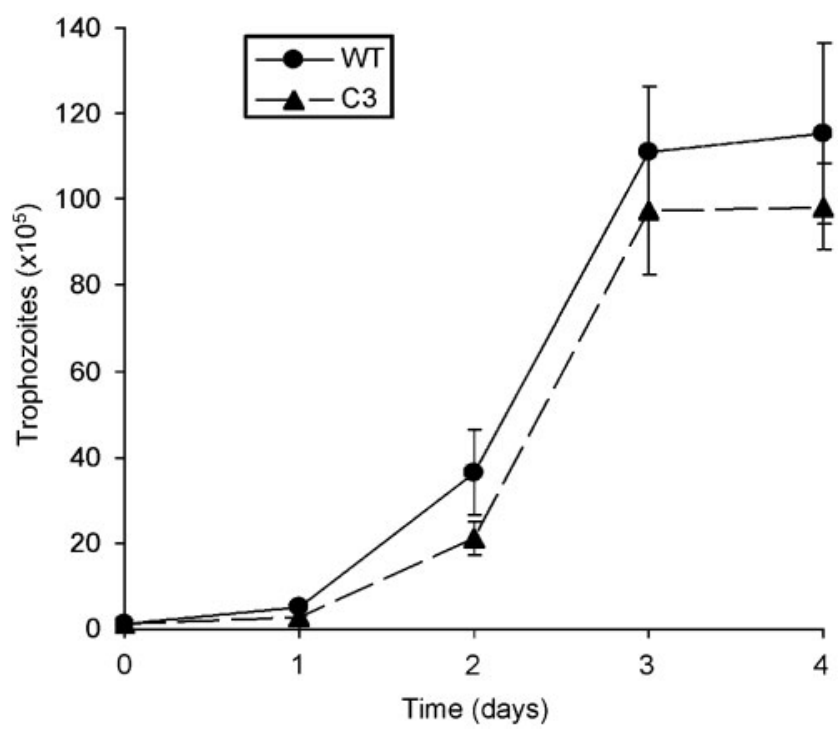

Fig. 2. Growth of Giardia lamblia WB C6 wild-type (WT) and FOR-resistant (C3) trophozoites. At day $0,10^{4}$ trophozoites were inoculated to normal culture medium and trophozoite numbers were determined daily during a period of 4 days. Trophozoite numbers per culture tube are given as mean values ( \pm SEM) from triplicate determinations.

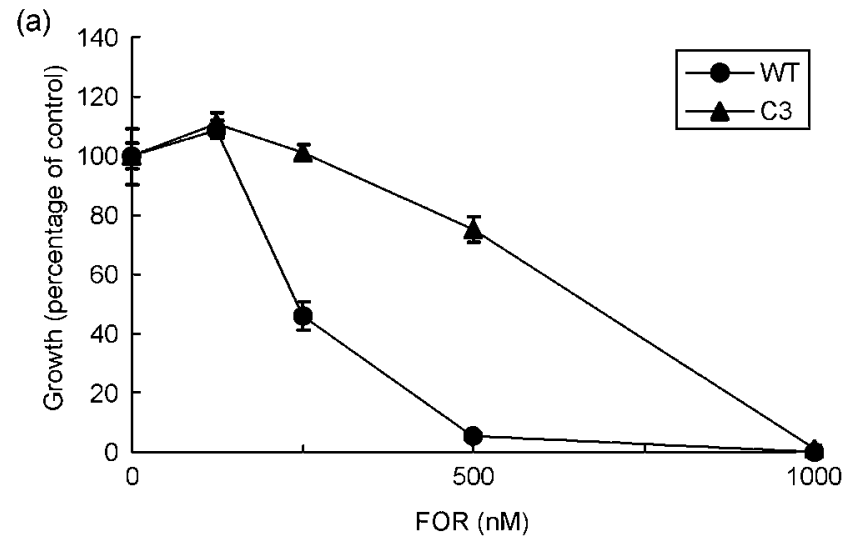

(b)

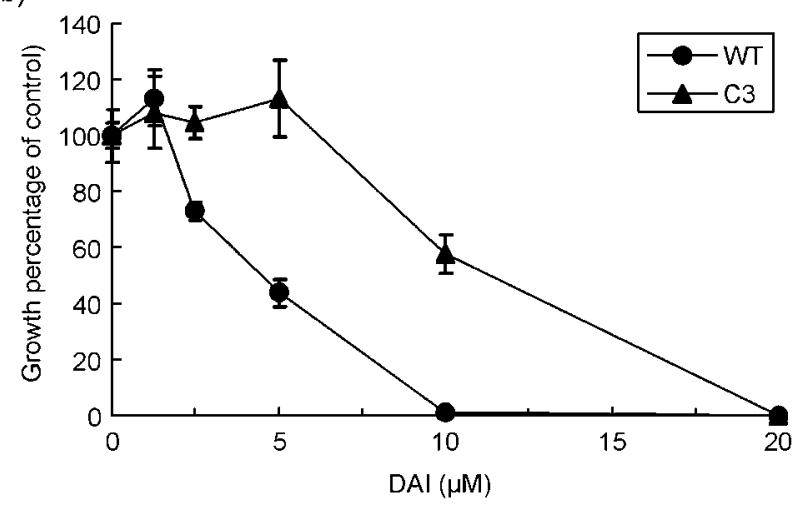

Fig. 3. Growth of Giardia lamblia WB C6 wild-type (WT) and isoflavone resistant (C3) trophozoites in the presence of formononetin (FOR, a) or daidzein (DAl, b). At day $0,10^{4}$ trophozoites were inoculated to normal culture medium. Trophozoites were harvested and counted at day 4 . Trophozoite numbers are given as mean values ( \pm SEM) from triplicate determinations.

at various drug concentrations (Fig. 3). At $0.5 \mu \mathrm{M}$ FOR, C3 had about $75 \%$ of control growth, whereas the wild-type was decreased to approximately $1.5 \%$ (Fig. 3a). On $10 \mu \mathrm{M}$ DAI, C3 reached approximately $58 \%$ of the control level whereas the wild-type was decreased to approximately $1 \%$ (Fig. 3b). Growth on GEN essentially corresponded to growth on DAI (growth curves not shown). C3 could thus be regarded as isoflavone-resistant.

In order to investigate whether resistance formation of C3 was a multidrug-resistance phenomenon, $\mathrm{C} 3$ and wild-type cells were grown in the presence of the commonly used antigiardial nitro drugs NTZ and MET. Both wild-type and C3 trophozoites were equally sensitive to these compounds (growth curves not shown).

\section{A nucleoside hydrolase homologue is a major DAl-binding protein in Giardia lamblia}

Following DAI-affinity chromatography, a major Giardia protein of approximately $40 \mathrm{kDa}$ was eluted with $0.1 \mathrm{mM}$ FOR (Fig. 4a, lane FOR). FOR was used as eluent since this 

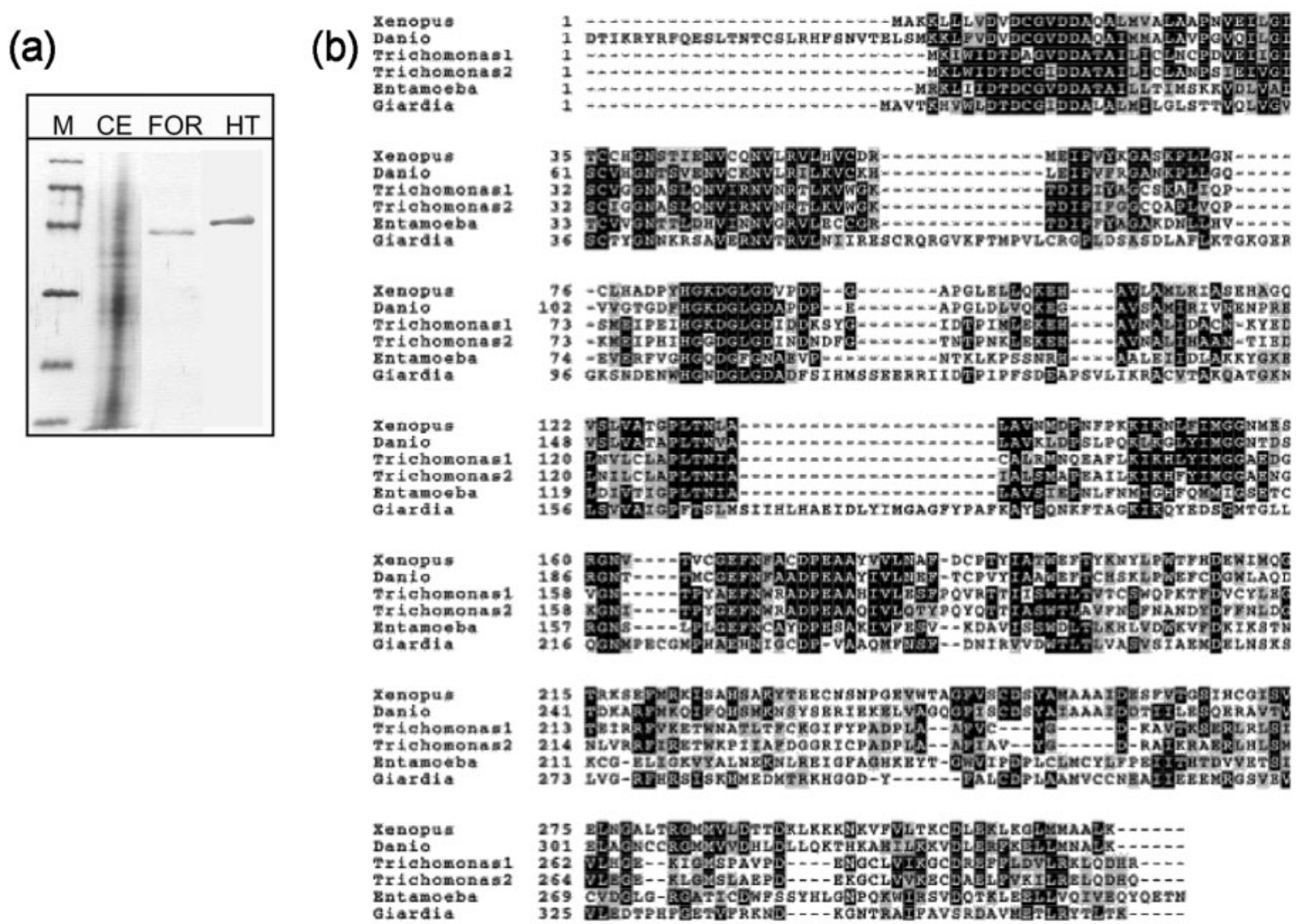

Fig. 4. (a) Affinity chromatography of cell-free extracts from $3 \times 10^{8}$ Giardia lamblia trophozoites. Aliquots of fractions were separated by SDS-PAGE and a silver staining of the gel was performed. M, marker proteins $(92,66,45,31,22.5$ and $14 \mathrm{kDa})$; $\mathrm{CE}$, crude extract; FOR, elution with FOR (0.5 mM in PBS); HT, recombinant EAA37551.1 eluted after His-tag affinity purification. (b) Alignment of EAA37551.1 (Giardia) with its closest homologues, the Trichomonas vaginalis nucleoside hydrolase EAY18059 (Trichomonas 1), the Trichomonas vaginalis inosine-uridine-preferring nucleoside hydrolase family protein (Trichomonas 2), the Xenopus tropicalis protein containing an inosine-uridine-preferring nucleoside hydrolase domain NP_001039191 (Xenopus), the Danio rerio LOC402865 protein AAH56692 (Danio) and the Entamoeba histolytica inosineuridine-preferring nucleoside hydrolase XP_654837 (Entamoeba). The alignment was produced by CLUSTAL W (Huang \& Miller, 1991).

compound showed the best growth effects on Giardia trophozoites and since the blocking of one $\mathrm{OH}$ group corresponded best to the ligand DAI bound via a C12spacer to one of its $\mathrm{OH}$ groups to the matrix. By mass spectrometry, the $40 \mathrm{kDa}$ protein was identified as a nucleoside hydrolase (NH) homologue (accession EAA37551.1; orf 13272 in the Giardia genome database; approximately $30 \%$ coverage of the sequence by peptides from MS sequencing). Protein-protein BLAST (Altschul et al., 1997) revealed that the closest homologues of EAA37551.1 were NHs from Trichomonas vaginalis (two enzymes), Entamoeba histolytica, Danio rerio and Xenopus tropicalis. Besides the conserved domains, EAA37551.1 possessed, however, short peptide motives (between aa residues 61 and 226) not found in the other NHs (Fig. 4b), a phenomenon that has been observed in many other Giardia orthologues (see Giardia genome database). Like its homologues, EAA37551.1 had no signal peptide and was considered as cytosolic.

\section{EAA37551.1 exhibits nucleoside hydrolase activity and is named GINH}

The coding sequence of EAA37551.1 was amplified by PCR and cloned and expressed in E. coli. The resulting product migrated at around $40 \mathrm{kDa}$ by SDS-PAGE. The recombinant protein was the major protein in IPTG-induced Escherichia coli and could be further purified by His-tag affinity chromatography (Fig. 4a). The purified protein was subjected to an indirect nucleoside hydrolase assay based on the determination of riboside released from various nucleosides. The recombinant protein hydrolysed the purine nucleosides adenosine, guanosine and inosine much better than the pyrimidine nucleosides uridine or cytidine (Fig. 5a). 

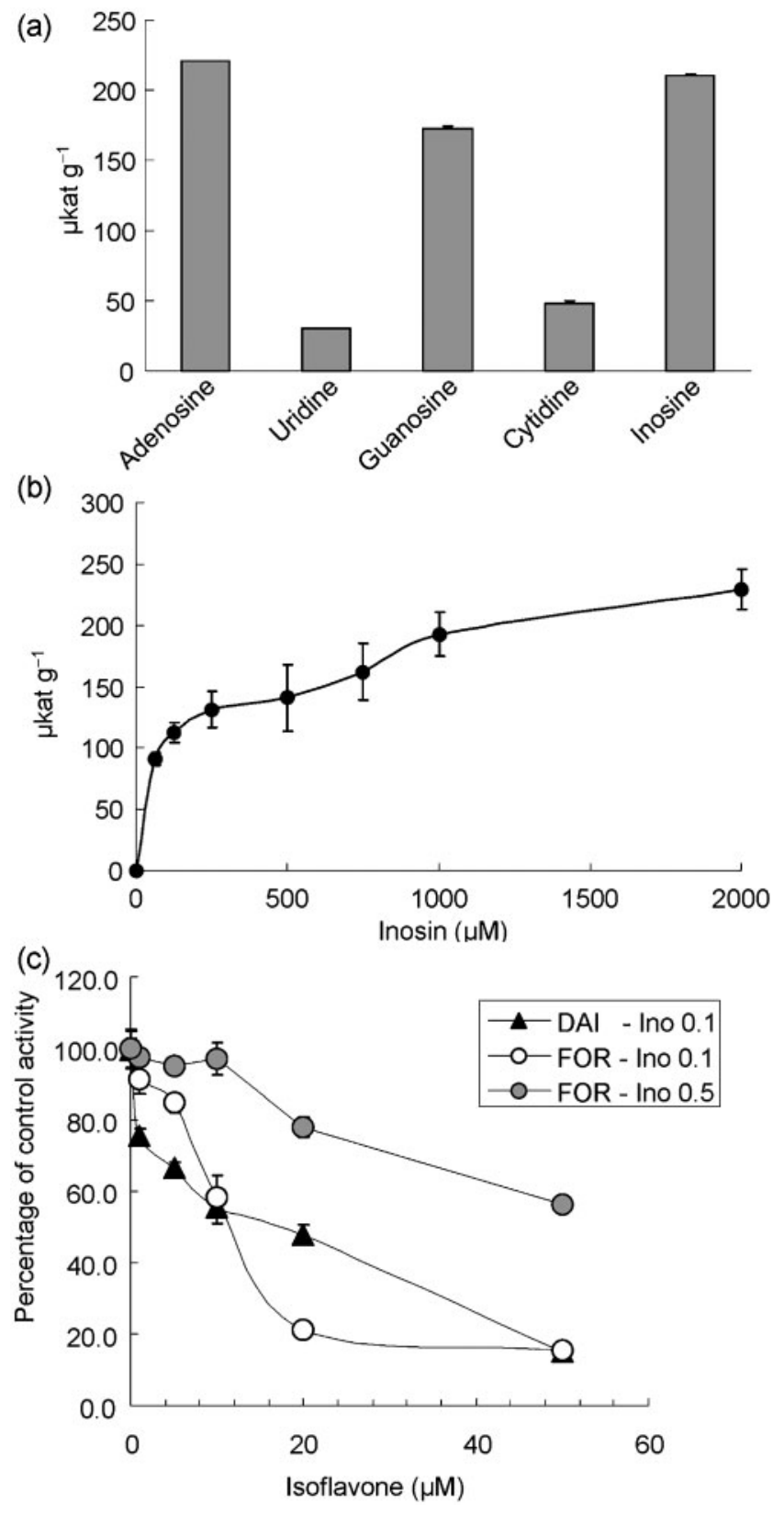

Fig. 5. Nucleoside hydrolase activity of EAA37551.1. (a) Activity on various nucleosides (2 $\mathrm{mM}$ each) was measured in an indirect assay system at $37{ }^{\circ} \mathrm{C}$. (b) Direct assay for $K_{\mathrm{m}}$ determination with various concentrations of inosine. (c) Indirect assay with inosine (Ino, 0.1 or $0.5 \mathrm{mM}$ ) as a substrate and the isoflavones daidzein (DAI) or formononetin (FOR) as inhibitors. Values correspond to triplicates (mean values \pm SEM).

In order to determine the kinetic constants of $\mathrm{GlNH}$, recGlNH was subjected to a direct enzyme assay using inosine as a substrate. In this system, the $K_{\mathrm{m}}$ value was $70 \pm 11.5 \mu \mathrm{M}$ and the $V_{\mathrm{m}}$ was $185 \pm 32 \mu \mathrm{kat} \mathrm{g}^{-1}$ as determined after a Lineweaver-Burk transformation of the curve shown in Fig. 5(b). These values were in good agreement with previously published values from NHs from Crithidia fasciculata (Parkin et al., 1991) and Trichomonas vaginalis Gero et al. (2000). Thus, we propose that EAA37551.1 should be named G. lamblia purine nucleoside-preferring nucleoside hydrolase $(\mathrm{GlNH})$.

\section{Recombinant nucleoside hydrolase (NH) is inhibited by isoflavones}

When an indirect $\mathrm{NH}$ assay was performed in the presence of DAI and FOR, NH activity decreased in a concentrationdependent manner. The inhibition was more pronounced at $0.1 \mathrm{mM}$ than at $0.5 \mathrm{mM}$ of the substrate inosine (Fig. 5c). GEN behaved similarly to DAI (data not shown). Assuming a competitive inhibition, the $K_{\mathrm{i}}$ values were $3.8 \pm 0.7 \mu \mathrm{M}$ for DAI and $11 \pm 2.8 \mu \mathrm{M}$ for FOR as calculated according to Parkin (1996).

\section{Gene expression}

In order to determine gene expression of $\mathrm{NH}$ identified as potential target of isoflavones (see above) and thus potentially involved in resistance formation, we developed a real-time RT-PCR assay quantifying reverse transcripts of the $\mathrm{NH}$ gene. In this assay, reverse transcripts of ACT gene were also quantified as reference for the amount of total cDNA. ACT gene expression had been previously revealed to be constant in WB C6 wild-type and drug-resistant strains (Müller et al., 2007). Respective analysis demonstrated that gene expression of $\mathrm{NH}$ was significantly decreased in C3 (approx. 41\%) compared with the corresponding wild-type WB C6 expression level (Fig. 6). However, the differences between the wild-type and the resistant G. lamblia clone were much more pronounced when expression of VSP genes was investigated. For respective analyses, cDNA was synthesized issuing from polyT-ANC primers (see Methods). This experimental strategy allowed RT-PCRs to be performed with a general forward primer (MM16 primer) targeted to a highly conserved VSP gene region and a reverse primer targeted to the $3^{\prime}$ terminal anchor (ANC) sequence adjacent to the polyT stretch of the cDNA molecules (Table 1). By comparative quantitative RT-PCR, the cDNA synthesized with polyT-ANC was also amplified by including a primer pair specific for the gene TSA417 that encodes the major VSP in WB C6 (Fig. 6). While wild-type clone WB C6 exhibited TSA417 gene expression, corresponding expression levels were dramatically reduced and virtually nondetectable in FORr clone C3. Conversely, the overall expression of VSP genes (VSPtot) remained constant, suggesting that resistance was associated with an antigenic switch from TSA417 to one, or several unknown, VSPs

\section{DISCUSSION}

In the past, various antiparasitic drugs have been developed but only a few compounds such as MET and NTZ turned 


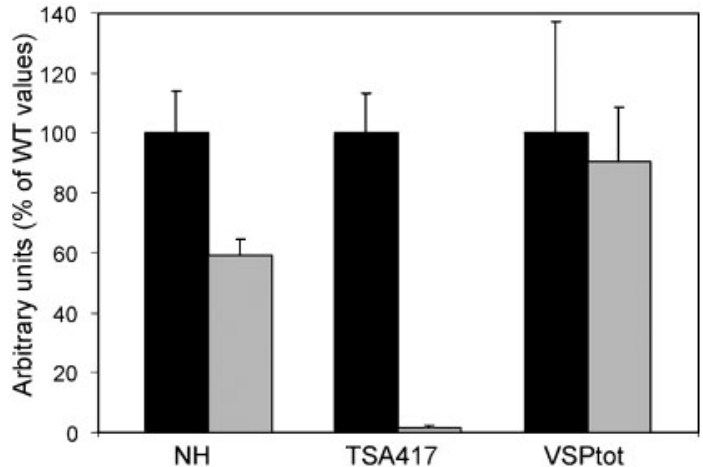

Fig. 6. Quantification of $\mathrm{NH}$ and variant surface protein (VSP) gene expression by real-time RT-PCR. Trophozoites were grown in normal culture medium until near confluency was reached. RNA was extracted and reverse transcribed to cDNA. Transcripts of $\mathrm{NH}$, VSP gene encoding TSA417, and of total VSPs (VSPtot) were quantified in relation to transcripts from constitutively expressed actin (ACT) gene. The relation is expressed in arbitrary units (AU) as a percentage of the wild-type (WT) mean values ( \pm SEM).

out to be effective for treatment of giardiasis (reviewed by Gardner \& Hill, 2001; Upcroft \& Upcroft, 2001). In the field of antigiardial drug development, the possibility of resistance formation has been recognized as an important problem and was the subject of various preclinical and clinical investigations (reviewed by Upcroft \& Upcroft, 2001). Formation of resistance to MET is a major constraint for efficient treatment of human giardiasis, and therapeutic failure due to giardial resistance to this compound is occurring at an increasing frequency (Townson et al., 1994; Wright et al., 2003). Furthermore, our recent investigations revealed that G. lamblia trophozoites grown in vitro were able to develop resistance to NTZ, and at least one of the resistant clones exhibited cross-resistance to MET (Müller et al., 2007).

Under the circumstances outlined above, the isoflavones previously described as a new group of antimicrobial compounds (reviewed by Dakora \& Phillips, 1996) have been investigated as alternative drugs for the treatment of giardiasis (Khan et al., 2000). Among the different isoflavones tested in vitro, FOR turned out to be the most efficacious antigiardial compound (Khan et al., 2000) and was also demonstrated to efficiently inhibit growth of MET- and/or NTZ-resistant G. lamblia strains (Müller et al., 2007). This observed lack of cross-resistance in vitro certainly suggested a further evaluation of FOR regarding its applicability as an alternative antigiardial agent to overcome potential problems of resistance to conventional drugs. Conversely, it is evident that this concept of treatment may be complicated by the capacity of Giardia to develop high-level resistance to this drug. In fact, in our study, resistance to FOR was already detectable upon relatively short exposure of in vitro-cultivated trophozoites to the drug, suggesting that genetic predispositions allow the parasite to immediately adapt to an isoflavone-enriched host intestinal environment, e.g. emerging upon uptake of an isoflavone-rich vegetarian diet by the host.

In the present study, trophozoites from FORr clone C3 were found to be cross-resistant to other isoflavones DAI and GEN, but not to the nitro compounds MET or NTZ. This indicated that resistance was not caused by a general mechanism like multi-drug transporters, but rather by an as yet unknown mechanism that specifically concerns isoflavones. This conclusion was also supported by our previous observation demonstrating that NTZ-resistant G. lamblia trophozoites were not cross-resistant to isoflavones (Müller et al., 2007).

One, but not the only, effect of isoflavones could be the inhibition of the isoflavone-binding protein $\mathrm{NH}$. This inhibition occurs in a range of concentrations where DAI and GEN inhibit trophozoite growth in vivo. FOR is inhibitory at much lower concentrations, suggesting other mechanisms to be involved. In Giardia, hydrolysis of exogenous nucleosides by $\mathrm{NH}$ has been previously described as the first step of one of two distinct salvage pathways to form purine (see e.g. Wang \& Aldritt, 1983; Aldritt \& Wang, 1986) and pyrimidine ribonucleotides (Aldritt et al., 1985; for review see Jarroll et al., 1989). Moreover, a low-affinity transporter for purine nucleobases has been described as a potential adenine and guanine donor for the second salvage pathway (Ey et al., 1992). In Crithidia, Leishmania or Trypanosoma, the function of $\mathrm{NH}$ is central in salvaging purine nucleosides from the host and essential since the parasites lack purine de novo synthesis (Parkin et al., 1991; Parkin, 1996; Shi et al., 1999). Inhibition of $\mathrm{NH}$ by a drug would thus lead ultimately to a block of DNA synthesis and thus cell division. Parasites could overcome this inhibition by synthesizing more $\mathrm{NH}$. An evaluation of the gene expression pattern revealed, however, a significant reduction of $\mathrm{NH}$ transcripts in the isoflavone-resistant clone C3 rather than an increase. This pattern is expected if the target enzyme activates a (nontoxic) prodrug, e.g. pyruvate-oxidoreductase in the case of MET (see Müller et al., 2007 and references therein). One explanation could be that $\mathrm{NH}$ is downregulated and another, unrelated scavenging enzyme such as purine phosphorylase (Parkin et al., 1991; Parkin, 1996) is upregulated, thus compensating $\mathrm{NH}$ inhibition.

In comparison to $\mathrm{NH}$ gene expression, the gene regulatory effects associated with antigenic variation of the parasite were much more pronounced. Here, expression of TSA417 representing the major surface antigen (VSP C6) of WB C6 was massively downregulated in isoflavone-resistant clone C3. This finding is in agreement with results from our recent study that demonstrated a striking reduction of the TSA417 gene expression in trophozoites that had developed resistance to NTZ and/or MET (Müller et al., 2007). It is known from current literature that G. lamblia is able to escape host immunological defence mechanisms by changing the expression of its surface antigens (reviewed by 
e.g. Müller \& Gottstein, 1998; Müller and von Allmen, 2005). Furthermore, it was found that, besides the immunological pressure, physiological factors such as intestinal proteases also act as driving forces for antigenic diversification of the parasite (Nash et al., 1991). Finally, we now realized that drug pressure is able to select for (a) new surface antigen variant(s) within a G. lamblia trophozoite population (Müller et al., 2007 and present study). Taking all these observations into account, antigenic variation seems to be part of a versatile adaptive mechanism that allows the parasite to respond to a broad spectrum of environmental stress factors. As reported recently, an epigenetic mechanism, probably including gene acetylation, seems to be responsible for antigenic switching of the parasite (Kulakova et al., 2006). Possibly, this epigenetic mechanism involves pleiotropic generegulatory effects that mediate, or at least participate in, antigiardial drug resistance formation. Future studies based on differential and functional genomics and proteomics approaches will reveal if surface antigen alterations per se or rather pleiotropic gene-regulatory processes associated with a surface antigen switch are responsible for in vitroinduced drug resistance in G. lamblia.

\section{ACKNOWLEDGEMENTS}

This study was supported by grants from the Swiss Secretariat for Education and Science (SBF, COST Action B22; grant No. SBF C05.0104; N. M. \& J.M.) and the Swiss National Science Foundation (grant No. 3100A0-112532/1; A. H.). J. M. was partially financially supported by the University of Berne.

\section{REFERENCES}

Aldritt, S. M. \& Wang, C. C. (1986). Purification and characterization of guanine phosphoribosyltransferase from Giardia lamblia. J Biol Chem 261, 8528-8533.

Aldritt, S. M., Tien, P. \& Wang, C. C. (1985). Pyrimidine salvage in Giardia lamblia. J Exp Med 161, 437-445.

Altschul, S. F., Madden, T. L., Schäffer, A. A., Zhang, J., Zhang, Z., Miller, W. \& Lipman, D. L. (1997). Gapped BLAST and PSI-BLAST: a new generation of protein database search programs. Nucleic Acids Res 25, 3389-3402.

Blum, H., Beier, H. \& Gross, H. J. (1987). Improved silver staining of plant proteins, RNA and DNA in polyacrylamide gels. Electrophoresis 8, 93-99.

Dakora, F. D. \& Phillips, D. A. (1996). Diverse functions of isoflavonoids in legumes transcend anti-microbial definitions of phytoalexins. Physiol Mol Plant Pathol 49, 1-20.

Dixon, R. A. \& Steele, C. L. (1999). Flavonoids and isoflavonoids - a good mine for metabolic engineering. Trends Plant Sci 4, 394-400.

Ey, P. L., Davey, R. A. \& Duffield, G. A. (1992). A low-affinity nucleobase transporter in the protozoan parasite Giardia intestinalis. Biochim Biophys Acta 1109, 179-186.

Gardner, T. B. \& Hill, D. R. (2001). Treatment of giardiasis. Clin Microbiol Rev 14, 114-128.

Gero, A. M., Kang, E. W., Harvey, J. E., Schofield, P. J., Clinch, K. \& Furneaux, R. H. (2000). Trichomonas vaginalis: detection of nucleoside hydrolase activity as a potential screening procedure. Exp Parasitol 94, 125-128.

Huang, X. \& Miller, W. (1991). A time-efficient, linear-space local similarity algorithm. Adv Appl Math 12, 337-357.

Jarroll, E. L., Manning, P., Berrada, A., Hare, D. \& Lindmark, D. G. (1989). Biochemistry and metabolism of Giardia. J Protozool 36, 190-197.

Keister, D. B. (1983). Axenic culture of Giardia lamblia in TYI-S-33 medium supplemented with bile. Trans $R$ Soc Trop Med Hyg 77, 487-488.

Khan, I. A., Avery, M. A., Burandt, C. L., Goins, D. K., Mikell, J. R., Nash, T. E., Azadegan, A. \& Walker, L. A. (2000). Antigiardial activity of isoflavones from Dalbergia frutescens bark. J Nat Prod 63, 1414-1416.

Kulakova, L., Singer, S. M., Conrad, J. \& Nash, T. E. (2006). Epigenetic mechanisms are involved in the control of Giardia lamblia antigenic variation. Mol Microbiol 61, 1533-1542.

Laemmli, U. K. (1970). Cleavage of structural proteins during the assembly of the head of the bacteriophage T4. Nature 227, 680-685.

Megraud, F., Occhialini, A. \& Rossignol, J.-F. (1998). Nitazoxanide: a potential drug for eradication of Helicobacter pylori with no crossresistance to metronidazole. Antimicrob Agents Chemother 42, 2836-2840.

Müller, N. \& Gottstein, B. (1998). Antigenic variation and the murine immune response to Giardia lamblia. Int J Parasitol 28, 1829-1839.

Müller, N. \& von Allmen, N. (2005). Recent insights into the mucosal reactions associated with Giardia lamblia infections. Int J Parasitol 35, 1339-1347.

Müller, J., Rühle, G., Müller, N., Rossignol, J. F. \& Hemphill, A. (2006). In vitro effects of thiazolides on Giardia lamblia WB clone C6 cultured axenically and in coculture with $\mathrm{CaCo} 2$ cells. Antimicrob Agents Chemother 50, 162-170.

Müller, J., Sterk, M., Hemphill, A. \& Müller, N. (2007). Characterization of Giardia lamblia WB C6 clones resistant to nitazoxanide and to metronidazole. J Antimicrob Chemother 60, 280-287.

Nash, T. E., Merritt, J. W., Jr \& Conrad, J. T. (1991). Isolate and epitope variability in susceptibility of Giardia lamblia to intestinal proteases. Infect Immun 59, 1334-1340.

Parkin, D. W. (1996). Purine-specific nucleoside N-ribohydrolase from Trypanosoma brucei brucei. J Biol Chem 271, 21713-21719.

Parkin, D. W., Horenstein, B. A., Abdulah, D. A., Estupiñán, B. \& Schramm, V. L. (1991). Nucleoside hydrolase from Crithidia fasciculata. Metabolic role, purification, specificity, and kinetic mechanism. J Biol Chem 266, 20658-20665.

Savioli, L., Smith, H. \& Thompson, A. (2006). Giardia and Cryptosporidium join the 'Neglected Diseases Initiative'. Trends Parasitol 22, 203-208.

Shi, W., Schramm, V. L. \& Almo, S. C. (1999). Nucleoside hydrolase from Leishmania major. J Biol Chem 274, 21114-21120.

Thompson, R. C. A. (2000). Giardiasis as a re-emerging infectious disease and its zoonotic potential. Int J Parasitol 30, 1259-1267.

Townson, S. M., Boreham, P. F., Upcroft, P. \& Upcroft, J. A. (1994). Resistance to the nitroheterocyclic drugs. Acta Trop 56, 173-194.

Upcroft, P. \& Upcroft, J. A. (2001). Drug targets and mechanisms of resistance in the anaerobic protozoa. Clin Microbiol Rev 14, 150-164.

Upcroft, J. A., Campbell, R. W., Benakli, K., Upcroft, P. \& Vanelle, P. (1999). Efficacy of new 5-nitroimidazoles against metronidazole-susceptible and -resistant Giardia, Trichomonas, and Entamoeba spp. Antimicrob Agents Chemother 43, 73-76.

Upcroft, J. A., Dunn, L. A., Wright, J. M., Benakli, K., Upcroft, P. \& Vanelle, P. (2006). 5-nitroimidazole drugs effective against metroni- 
dazole-resistant Trichomonas vaginalis and Giardia duodenalis. Antimicrob Agents Chemother 50, 344-347.

von Allmen, N., Bienz, M., Hemphill, A. \& Müller, N. (2004). Experimental infections of neonatal mice with cysts of Giardia lamblia clone GS/M-83-H7 are associated with an antigenic reset of the parasite. Infect Immun 72, 4763-4771.
Wang, C. C. \& Aldritt, S. (1983). Purine salvage networks in Giardia lamblia. J Exp Med 158, 1703-1712.

Wright, J. M., Dunn, L. A., Upcroft, P. \& Upcroft, J. A. (2003). Efficacy of antigiardial drugs. Expert Opin Drug Saf 2, 529-541.

Edited by: J. Tachezy 